\title{
Questes
}

\section{Secret, public, privé. Quelques pistes de réflexion}

\section{Clémence Revest}

\section{(2) OpenEdition}

\section{Journals}

\section{Édition électronique}

URL : http://journals.openedition.org/questes/4175

DOI : 10.4000/questes.4175

ISSN : 2109-9472

\section{Éditeur}

Les Amis de Questes

\section{Édition imprimée}

Date de publication : 15 avril 2009

Pagination : 1-11

ISSN : 2102-7188

\section{Référence électronique}

Clémence Revest, «Secret, public, privé. Quelques pistes de réflexion », Questes [En ligne], 16 | 2009,

mis en ligne le 20 juin 2015, consulté le 23 septembre 2020. URL : http://journals.openedition.org/ questes/4175; DOI : https://doi.org/10.4000/questes.4175 


\section{Secret, public, privé. \\ Quelques pistes de réflexion}

\section{Clémence REVEST}

Ces courtes remarques introductives prendront pour point de départ le premier mot proposé, celui de « secret». Cependant, que l'on ne s'y trompe pas, il ne s'agit pas seulement de respecter l'ordre du libellé tel qu'énoncé mais bien de souligner d'emblée l'idée que de cette notion peut découler la définition des deux autres termes, ceux de «public » et «privé », beaucoup plus couramment utilisés par les chercheurs, figés ou galvaudés parfois. Le problème que ce thème sous forme de triptyque invite à développer consisterait ainsi avant toute chose dans l'affirmation selon laquelle le secret peut être considéré comme un instrument de réflexion pertinent pour penser la frontière entre public et privé au Moyen Âge.

En effet, l'insertion du terme «secret» au sein de ce qui est habituellement un duo antagoniste doit être comprise comme une incitation à repenser la définition de ces deux sphères en termes dynamiques, c'est-à-dire pas seulement comme des espaces à la fois séparés et concomitants, à la manière de cercles concentriques qui s'emboîteraient, délimités par la borne des degrés de sociabilité et par le seuil de la demeure domestique, mais comme les formes de l'appréhension individuelle de l'autre, de l'étranger et de l'étrange aux moyens de codes sociaux et culturels. Le secret apparaîtrait, dans cette perspective, comme une limite mouvante, manipulée, créée et possiblement transgressée entre l'homme, la société dans laquelle il vit, et le divin. 
Pour avancer ces premières hypothèses, il est possible de s'appuyer sur un certain nombre de travaux de recherche tout à fait récents qui ont choisi d'utiliser la notion de secret afin de rendre compte de l'interpénétration complexe du public et du privé dans la société et la littérature médiévales, et de ses enjeux conceptuels. De fait, celui qui se plongera plus attentivement dans des lectures évoquant cette question aura la bonne surprise de constater combien elle a fait l'objet dernièrement de travaux de grande qualité.

Avant de livrer quelques éléments plus précis concernant ces études, il est nécessaire de rappeler l'existence de deux développements théoriques poussés concernant le secret, parus dans les années 1970, et qui demeurent des points de repères fondamentaux. Il s'agit de l'ouvrage philosophique de Pierre Boutang, Ontologie du secret, tiré de sa thèse et paru en $1973^{1}$, et de l'article d'Arnaud Lévy paru en 1976 dans la Nouvelle Revue de psychanalyse et intitulé «Évaluation étymologique et sémantique du mot "secret" » - on aura bien évidemment compris que cette réflexion s'est inscrite dans le cadre du développement de la pratique psychanalytique en France, qui a fait des secrets de famille, du non-dit, du refoulement, du tabou, des catégories essentielles de sa pensée ${ }^{2}$.

Tous deux ont mis en lumière le caractère structurant du secret dans la relation sociale. Arnaud Lévy, par exemple, est parti d'une analyse étymologique : secret vient du latin secretum, qui est lui-même le participe passé du verbe secerno. Ce verbe est formé d'un préfixe, -se, qui indique la séparation, et du radical -cerno qui signifie trier, distinguer. Dans son premier sens, ce verbe désignait le fait de tamiser le gravier, de séparer le bon grain de

\footnotetext{
${ }^{1}$ BoutAng, Pierre, Ontologie du Secret, Paris, PUF, Quadrige, 1973.

2 LEVY, Arnaud, "Évaluation étymologique et sémantique du mot "secret"», Nouvelle revue de psychanalyse, 14 (1976), p. 117-129.
} 
l'ivraie. Un premier sème s'imposait donc, celui de la séparation. De là pouvait être posée la question du contenu, difficulté que l'auteur résout avec beaucoup de clarté :

«Les dictionnaires font état de secrets très divers, de nature très différente. Comme secret, on trouve aussi bien des éléments de psychisme (pensées, désirs, sentiments, desseins), de comportement (négociations, intrigues, recettes de fabrication), que des objets matériels (tiroirs, mécanismes de serrures, portes, escaliers, lieux). Tout semble pouvoir être un secret, même rien. Mais le polymorphisme des secrets n'est qu'apparent, car tout se ramène en dernier lieu à un savoir. C'est le savoir de la chose, et non la chose elle-même, qui constitue le secret. $)^{3}$

Nous disposons dès lors d'un deuxième sème, celui du savoir, qui peut s'appliquer à toute chose, telle que la médecine, la politique, la famille, la science, ou encore le divin. Mais, pour donner son sens complet au mot secret, il a fallu faire intervenir un troisième sème qui en était comme la raison d'être, c'est-à-dire autrui, ou plus précisément une relation à un autre (collectif ou individuel) construite autour de ce savoir dissimulé.

C'est pourquoi il y a nécessairement un dépositaire du secret - celui qui sait - mais aussi un dépositaire «paradoxal », c'est-à-dire celui qui en est exclu. Ainsi le secret permet-il de structurer les relations sociales en termes de domination (entre initié et profane, espion et victime par exemple), mais aussi en tant que fondement de paix et de consensus, car il peut contribuer au nécessaire maintien d'une fiction collective.

\footnotetext{
${ }^{3}$ LEVY, Arnaud, art. cit., p. 120. Frédérique Le Nan cite ce même passage dans son ouvrage présenté plus loin.
} 
Quittant le domaine de l'abstraction, nous remarquerons que les travaux en critique littéraire et en histoire portant sur cette question ont affiné ces pistes de réflexion en insistant tout d'abord sur les fortes variations du champ sémantique lié au secret (clandestin, occulte, confidentiel, opaque etc.). Deux pistes de recherche en sont globalement ressorties: d'une part, l'ambiguïté morale du secret et du discours qui s'y rattache, d'autre part sa fonction dramatique et imaginaire.

Dans le domaine de la littérature, un grand nombre d'études extrêmement stimulantes sont parues sur le thème du secret, dépassant largement le champ du Moyen Âge. De manière générale, je retiendrai les actes du Colloque tenus à Louvain-la-Neuve en mars 1996, et publiés en 1999 sous la direction de Chantal Zabus, sous le titre Le Secret : motif et moteur de la littérature ${ }^{4}$.

La littérature médiévale n'est pas en reste dans ce domaine, bien au contraire, et l'on ne peut que constater le foisonnement et la qualité des travaux, parus depuis les années 1990, consacrés au rôle du secret dans la littérature médiévale.

Deux, surtout, doivent retenir notre attention. Le plus récent et le plus important probablement est l'ouvrage de Frédérique Le Nan, tiré de sa thèse soutenue à l'Université de Montpellier III en 1996 sous la direction de Francis Dubost, Le Secret dans littérature narrative arthurienne (1150-1250). Du lexique au motif ${ }^{5}$. Soulignons d'abord que cet ouvrage clair et accessible se lit avec beaucoup de plaisir. Pour en venir plus précisément au contenu de cette étude, on retiendra le fait que Frédérique Le Nan identifie trois vocables

\footnotetext{
${ }^{4}$ ZABUS, Chantal (dir.), Le Secret : motif et moteur de la littérature, Louvain-la-Neuve, Collège Érasme, 1999.

${ }^{5}$ LE NAN, Frédérique, Le Secret dans littérature narrative arthurienne (1150-1250). Du lexique au motif, Paris, Champion, 2002.
} 
majeurs, dans son corpus, qui sont liés au champ du secret : celer (le plus représenté), secroi et conseil (repostailles est également relevé). L'auteur montre, partant de là, que le terme celer (avec conseil) a trait au secret dans le monde humain, terrestre, tandis que l'usage du terme secroi semble réservé à l'ordre divin, supérieur, et particulièrement au thème du Graal ${ }^{6}$. Frédérique Le Nan identifie ensuite, en ce qui concerne le secret humain, trois grands motifs qui sont les tares secrètes, les naissances illégitimes, et les amours interdites ${ }^{7}$.

Dans l'optique qui nous occupe, il est tout à fait intéressant de remarquer combien l'auteur a essayé, au cours de son analyse, de définir le roman médiéval lui-même comme un espace du secret ${ }^{8}$. Le secret y est compris comme clé des ressorts dramatiques, en tant qu'élément actanciel fondamental, et il est également envisagé au travers de la fonction cathartique qu'il assigne au roman. Écrire ce qui doit en principe être tu, livrer le caché à la connaissance de tous, reviendrait dans ce sens à accomplir une forme de transgression sociale.

Un second ouvrage représentatif de ces pistes de réflexions, plus ancien, est celui de Anthony Colin Spearing, paru en 1993, qui s'intitule The Medieval Poet as Voyeur: Looking and Listening in Medieval LoveNarratives ${ }^{9}$. L'auteur tente d'y démontrer, au travers d'une suite d'exemples, comment, dans le récit d'amour médiéval, le lecteur est invité à briser les secrets, à regarder en voyeur, à espionner l'interdit.

\footnotetext{
${ }^{6}$ Ibid., p. $29-58$.

${ }^{7}$ Frédérique Le Nan y consacre entièrement sa troisième et dernière partie.

${ }^{8}$ Ibid., p. 95-105.

9 Spearing, Anthony Colin, The Medieval Poet as Voyeur: Looking and Listening in Medieval Love-Narratives, Cambridge, New York, Cambridge University Press, 1993.
} 
La recherche historique, en revanche, s'est dans un premier temps moins intéressée directement à la notion de « secret », du moins d'un point de vue strictement quantitatif. Ce retard peut, me semble-t-il, trouver des éléments d'explication dans le très fort impact qu'a eue l'histoire de la vie privée conçue comme une histoire de la famille, autrement dit fondée sur une définition de la sphère privée comprise comme la diversité des formes de la vie sociale dans le cadre d'une maison et d'une parenté. C'est bien ainsi que Georges Duby définissait la notion de privacy dans l'introduction du tome 2 de l'Histoire de la vie privée, De l'Europe féodale à la Renaissance, parue en 1985 : une histoire de la vie privée qui serait d'abord une histoire de la convivialité, caractérisée par des lieux (la maison, le lit) et par des moments de sociabilité (le mariage, les fêtes) ${ }^{10}$. Il est patent que le problème, par exemple, du rapport de l'individu à ce système social ainsi que le thème du secret ne soient appréhendés qu'au dernier chapitre, qui définit la Renaissance comme le moment de la naissance de l'individualisme bourgeois.

Cependant, depuis plusieurs années, l'historiographie contemporaine a développé de nouvelles pistes de réflexion autour des rapports entre public et privé, qui placent au premier rang de leurs préoccupations la notion de « secret», en tant qu'élément central de définition. Ces travaux de recherche vont de pair avec des problématiques juridiques apparues récemment sur le devant de la scène, dont trois sont prégnantes :

1. La question de la mémoire collective, et de son corollaire, le refoulement, avec toute la dimension psychologique qui l'accompagne, c'està-dire la honte, l'oubli volontaire, le mensonge.

\footnotetext{
${ }^{10}$ DuBy, Georges, ARIES, Philippe (dir.), Histoire de la vie privée. 2. De l'Europe féodale à la Renaissance, Paris, Seuil, 1985.
} 
2. La question du secret d'État ensuite, liée à la mise en place des services de renseignement et d'espionnage. Il peut s'agir également, dans la même perspective, de secret industriel ou financier.

3. La question de la protection de la vie privée enfin, face à un développement des médias qui tend à rendre confuses les frontières entre vie privée et vie publique.

Deux éléments de cette production historiographique peuvent encore une fois retenir tout particulièrement notre attention. Premièrement, il s'agit de l'ouvrage d'Alain Dewerpe paru en 1994 sous le titre Espion. Une anthropologie historique du secret d'État contemporain ${ }^{11}$, dans lequel l'auteur essaie de dépasser ce qui semble être au départ une «immobile et invariante nature du politique $»^{12}$. Deuxièmement, nous pouvons nous référer au long chapitre écrit par Gérard Vincent dans le dernier de l'Histoire de la vie privée, qui porte un titre significatif : «Une histoire du secret ? $»^{13}$.

Les deux auteurs se rejoignent notamment sur un point déjà brièvement évoqué, à savoir les contradictions du discours moral autour de l'usage du secret. Le terme lui-même change de connotation selon qu'il désigne ou non une pratique de rétention de l'information considérée comme légitime. En effet, le secret peut être pris dans un sens très péjoratif, celui de la clandestinité, de la trahison, du complot, de la malhonnêteté ; à partir de là, est construit un discours idéologique de la transparence, de la nécessité de publicité. Mais à l'inverse, le secret peut être pris dans un sens licite, celui de

${ }^{11}$ DEWERPE, Alain, Espion. Une anthropologie historique du secret d'État contemporain, Paris, Gallimard, 1994.

${ }^{12}$ Ibid., p. 11.

13 VINCENT, Gérard, «Une histoire du secret? », in Georges DUBY, Philippe ARIES (dir.), Histoire de la vie privée. 5. De la première guerre mondiale à nos jours, Paris, Seuil, 1987, p. 133-250. 
la nécessité politique, de l'exigence de discrétion, du respect d'autrui ; à partir de là est construit un discours qui appelle à la sécurité de l'anonymat et à la protection des intérêts de la collectivité. Le vocabulaire est révélateur de ce double penchant, puisqu'un secret peut être aussi bien violé, brisé, trahi, que révélé, dévoilé ou simplement ébruité, divulgué.

Ces constats doivent nous inciter à nous interroger sur les modes de légitimation des pratiques de rétention de l'information, sur leurs moyens, leurs fondements éthiques ou juridiques, et leurs conséquences politiques. Mais il faut aussi se demander dans quelle mesure nous sommes capables d'en faire un objet d'enquête historique, partant de ce paradoxe qui voudrait que l'on cherche à connaître ce qui, par principe, aurait dû rester ignoré.

Je rejoindrai sur cette question les démarches amorcées par les chercheurs en littérature, et j'insisterai au contraire sur la portée dynamique du secret, comme étant précisément le déclencheur de l'enquête et un appel lancé à l'imagination de l'historien. Sans mystère, pas d'investigation. Rappelons aussi, dans cette perspective, que c'est par le secret que les fantasmes collectifs autour de l'histoire se développent, par exemple autour des « secrets des Templiers ».

Plus sérieusement, la notion de secret est également nécessaire si l'on veut comprendre les rapports entre privé et public à partir de l'individu, en tant qu'il s'intègre à un système social, politique et culturel. On voudrait en ce sens mettre au jour les barrières qu'il dresse entre lui et ces mêmes systèmes et qu'au besoin il transgresse.

L'historiographie médiévale s'est également emparée de cette piste de recherche depuis un vingtaine d'années. Peter Von Moos et Jacques Chiffoleau en particulier ont notablement approfondi les pistes de réflexion 
autour du secret, en s'intéressant notamment au problème de la conscience de l'individu face à l'institution ecclésiale ${ }^{14}$.

Très récemment la revue Micrologus a pu ainsi, au travers de son dernier numéro paru en 2006 et intitulé $\mathrm{Il}$ segreto, fournir un panorama tout à fait stimulant de la production féconde des médiévistes en la matière ${ }^{15}$. Parmi les dix-sept articles présentés, une très grande majorité porte sur des thèmes liés à des questions religieuses, ésotériques ou philosophiques (pour en citer quelques-uns pêle-mêle : la prédestination, la magie, l'alchimie, la confession, les mystères de la nature etc.). On a ainsi cherché à mieux comprendre, par ce biais, la manière dont l'homme médiéval avait pu utiliser le secret pour penser son rapport à Dieu, à l'âme et à la nature, et ce, en insistant sur les différents usages des occulta cordis et des arcana dei. Le secret, notamment, permettrait d'interpréter l'inconnu, et pour reprendre l'expression utilisée par Nicolas Weill-Parot dans son article portant sur le recours à l'occulte dans les sciences scolastiques de la nature ${ }^{16}$, il s'agirait de «combler le vide dans l'explication $»^{17}$. Comme en littérature, le secret aurait ainsi une portée structurante, en ce qu'il permet de supposer un savoir caché face à ce qui en apparence est inintelligible.

Ces communications portent cependant aussi pour certaines sur le versant politique du secret et soulignent les différents aspects de l'ambiguïté décrite plus haut, entre développement d'une éthique de la parole, de la non-

${ }^{14}$ Consulter par exemple : CHIFFOLEAU, Jacques, «Dire l'indicible. Remarques sur la catégorie du nefandum du XII ${ }^{\mathrm{e}}$ au XV $\mathrm{XV}^{\mathrm{e}}$ siècle », Annales E.S.C., (mars-avril 1990), p. 289324 ; Von Moos, Peter, «Occulta cordis. Contrôle de soi et confession au Moyen Âge », Médiévales, 29 (1995), p. 131-140 et 30 (1996), p. 117-137.

${ }^{15}$ Micrologus. Natura, Scienze e Società Medievali, XIV, Il segreto (2006).

${ }^{16}$ WeILl-PARot, Nicolas, «Encadrement ou dévoilement. L'occulte et le secret dans la nature chez Albert le Grand et Roger Bacon », Micrologus, op. cit., p. 151-170.

${ }^{17}$ Ibid., p. 153. 
dissimulation, et construction d'une idéologie de la ruse politique ${ }^{18}$. Pensons en particulier à la figure du secrétaire, dépositaire des secrets du gouvernant et pion central d'une politique fondée sur la confidentialité.

Enfin, au cours du long article qu'il a consacré au problème du secret dans les pratiques de la juridiction ecclésiastique, en partant de l'adage «Ecclesia de occultis non iudicat ${ }^{19}$, Jacques Chiffoleau s'est livré à une réflexion approfondie concernant les enjeux de cette question et a mis en lumière la difficulté, pour les hommes du Moyen Âge, à séparer distinctement ce qui relève du public de ce qui relève du privé, le problème de la révélation des secrets dans un cadre juridique étant posé, précisément. Il y fait en ce sens une distinction fondamentale entre le secret qui se rattache au secretum et le secret qui se rattache aux occulta. Le secretum fait référence à un savoir humain volontairement dissimulé, il a trait à l'ouïe, et, outre l'intimité du monde intérieur, il concerne généralement les stratégies que le politique construit vis-à-vis du contrôle de l'information :

«Beaucoup plus qu'il ne s'oppose au public en effet, le cœur secret du pouvoir reste ce lieu central à partir duquel l'espace public semble souvent se construire et se déployer, en se fondant sur cette séparation même. Au cœur du gouvernement des sujets, des ordres religieux, des familles, il est sans cesse requis des conseillers des souverains, des membres des chapitres délibérants, des parents ou des amis. ${ }^{20}$

Les occulta en revanche, qui ont trait au domaine sensible du regard, se réfèrent à des forces supérieures, à des réalités dépassant la compréhension

\footnotetext{
${ }^{18}$ Voir en particulier : ARTIFONI, Enrico, « Segreti e amicizie nell' educazione civile dell' età dei comuni », ibid., p. 259-273 ; JUCKER, Michael, « Secrets and Politics : Methodological and Communicationnal Aspects of Late Medieval Diplomacy », ibid., p. 275-310; PARAVICINI BAgliani, Agostino, «Bonifazio VIII e il Segreto », ibid., p. 311-322.

19 Chiffoleau, Jacques, "Ecclesia de occultis non iudicat”? L'Église, le Secret, l'Occulte, du XII ${ }^{\mathrm{e}}$ au XV $\mathrm{XV}^{\mathrm{e}}$ siècle », ibid., p. 359-481.

${ }^{20}$ Ibid., p. 361.
} 
«qui rappellent à l'homme sa finitude et ses limites ${ }^{21}$. Jacques Chiffoleau s'est interrogé ensuite sur l'apparente contradiction entre, d'une part, le développement du for interne (une zone exempte de toute juridiction, placée sous le seul regard de Dieu) à partir du XII ${ }^{\mathrm{e}}$ siècle, et, d'autre part, le développement de la pratique judiciaire qui porte en elle une exigence de publicité de la conscience : les développements casuistiques complexes qui en sont nés, permettant par exemple exceptionnellement la levée du sceau de la confession, ont alors été analysés comme autant de réponses apportées à des tensions touchant, fondamentalement, à la question l'autonomie de l'individu, entre rébellion et obéissance.

$\mathrm{Au}$ fil de ces lectures et de ces interrogations, du clandestin à l'intime, de l'inaccessible au tabou, du complot à la stratégie de représentation, le secret n'a cessé de se présenter comme la clé de définition des clivages construits, subis parfois, ou encore transgressés, objets de jeux et de politiques, entre le public et le privé, entre ces différentes strates mêlées qui définissent l'identité individuelle. En effet, il semble, au terme de ces quelques pistes de réflexion qui ne prétendent en rien à l'exhaustivité, ni bibliographique ni problématique, que le secret est d'abord une quête de création de sens et qu'il s'inscrit dans une logique de civilisation : définir le savoir que nous possédons, celui que nous nous résignons à ne pas posséder, et celui dont nous pouvons contrôler la divulgation signifierait en effet limiter nos sphères d'appartenance, accepter un ordre supérieur qui nous domine, et trouver sa place dans un monde.

${ }^{21}$ Ibid., p. 362. 